PROCEEDINGS OF THE

AMERICAN MATHEMATICAL SOCIETY

Volume 136, Number 6, June 2008, Pages 2007-2018

S 0002-9939(08)09043-6

Article electronically published on February 12, 2008

\title{
ON THE CONVERGENCE IN CAPACITY ON COMPACT KAHLER MANIFOLDS AND ITS APPLICATIONS
}

\author{
PHAM HOANG HIEP
}

(Communicated by Mei-Chi Shaw)

\begin{abstract}
The main aim of the present note is to study the convergence in $C_{X, \omega}$ on a compact Kahler mainfold $X$. The obtained results are used to study global extremal functions and describe the $\omega$-pluripolar hull of an $\omega$-pluripolar subset in $X$.
\end{abstract}

\section{INTRODUCTION}

The convergence in the capacity $C_{n}$ on domains in $\mathbf{C}^{n}$ introduced by Bedford and Taylor (see [BT2]) was investigated by Xing and Cegrell (see [Xi1], [Xi2], Ce3]). Recently Kołodziej (see [Ko2]) introduced the capacity $C_{X, \omega}$ on a compact Kahler manifold $X$. Next Guedj and Zeriahi studied it in GZ. They proved that $C_{X, \omega}$ is locally equivalent to $C_{n}$. The main aim of the present note is to study the convergence in $C_{X, \omega}$ on $X$. The obtained results are used to study global extremal functions and describe the $\omega$-pluripolar hull of an $\omega$-pluripolar subset in $X$. In section 2, we introduce a characterization of the convergence in $C_{X, \omega}$ of a sequence of $\omega$-plurisubharmonic functions on $X$. Next we prove under some conditions that the convergence in $C_{X, \omega}$ on $X$ implies the one in $C_{S, \omega \mid S}$ where $S$ is a smooth hypersurface in $X$. By applying this result, in section 3 we prove that if $E$ is an $\omega$-pluripolar set in $X \backslash S$ where $S$ is a smooth hypersurface in $X$, then $E_{X}^{*} \cap S$ is also $\omega_{S}$-pluripolar in $S$, where $E_{X}^{*}$ denotes the pluripolar hull of $E$.

For the general definition of the complex Monge-Ampère operator we refer the reader to the papers $\mathrm{BT} 1, \mathrm{BT} 2, \mathrm{Ce} 1, \mathrm{Ce} 2$.

\section{Preliminaries}

1.1. Let $X$ be a compact Kahler manifold with a fundamental form $\omega=\omega_{X}$ with $\int_{X} \omega^{n}=1$. An upper semicontinuous function $\varphi: X \rightarrow[-\infty,+\infty)$ is called $\omega$ plurisubharmonic $\left(\omega\right.$-psh) if $\omega+d d^{c} \varphi \geq 0$. $\operatorname{By} \operatorname{PSH}(X, \omega)\left(\operatorname{resp} \operatorname{PSH}^{-}(X, \omega)\right)$ we denote the set of $\omega$-psh (resp. negative $\omega$-psh) functions on $X$.

Received by the editors September 30, 2006 and, in revised form, December 11, 2006.

2000 Mathematics Subject Classification. Primary 32W20; Secondary 32 Q15.

Key words and phrases. Complex Monge-Ampère operator, $\omega$-plurisubharmonic functions, compact Kahler manifold.

This work is supported by the National Research Program for Natural Sciences, Vietnam.

(C)2008 American Mathematical Society

Reverts to public domain 28 years from publication 2007 
1.2. In [Ko2], Kołodziej introduced the capacity $C_{X, \omega}$ on $X$ by

$$
C_{X}(E)=C_{X, \omega}(E)=\sup \left\{\int_{E} \omega_{\varphi}^{n}: \varphi \in \operatorname{PSH}(X, \omega),-1 \leq \varphi \leq 0\right\}
$$

where $\omega_{\varphi}^{n}=\left(\omega+d d^{c} \varphi\right)^{n}$ and $n=\operatorname{dim} X$.

In [GZ, Guedj and Zeriahi proved that $C_{X}$ is a Choquet capacity on $X$ and

$$
C_{X}(E)=\int_{X}\left(-h_{E, \omega}^{*}\right) \omega_{h_{E, \omega}^{*}}^{n}
$$

where $h_{E, \omega}^{*}$ denotes the upper semicontinuous regularization of $h_{E, \omega}$ given by

$$
h_{E, \omega}(z)=\sup \left\{\varphi(z): \varphi \in \operatorname{PSH}^{-}(X, \omega),\left.\varphi\right|_{E} \leq-1\right\} .
$$

1.3. Let $u_{j}, u \in \operatorname{PSH}(X, \omega)$. We say that $\left\{u_{j}\right\}$ converges to $u$ in $C_{X}$ if

$$
C_{X}\left(\left\{\left|u_{j}-u\right|>\delta\right\}\right) \rightarrow 0
$$

as $j \rightarrow \infty$, for all $\delta>0$.

1.4. Let $S$ be a smooth hypersurface in $X$. For each $z \in S$ we find a neighbourhood $U$ of $z$ and a strictly psh function $\varphi$ on $U$ such that $\omega=d d^{c} \varphi$. Define $\left.\omega\right|_{S}=d d^{c} \varphi$ on $U \cap S$. Then $\omega_{S}$ is a fundamental form on $S$. Obviously if $u \in \operatorname{PSH}(X, \omega)$, then $\left.u\right|_{S} \in \operatorname{PSH}\left(S, \omega_{S}\right)$.

1.5. Let $E \subset X$. We say that $E$ is $\omega$-pluripolar if there exists $\varphi \in \operatorname{PSH}(X, \omega)$, $\varphi \not \equiv-\infty$ such that $E \subset\{\varphi=-\infty\}$. In GZ the authors proved that $E$ is $\omega$ pluripolar if and only if $E$ is locally pluripolar. Define

$$
E_{X}^{*}=\bigcap\{u=-\infty: u \in \operatorname{PSH}(X, \omega), u=-\infty \text { on } E\} .
$$

The set $E_{X}^{*}$ is called the $\omega$-pluripolar hull of $E$ in $X$.

\section{A characterization of COnVergence in $C_{X}$}

In this section we prove the following.

2.1. Theorem. Let $u_{j}, u \in P S H(X, \omega)$ be uniformly bounded. Then the following two are equivalent:

i) $u_{j} \rightarrow u$ in $C_{X}$;

ii) $\varlimsup_{j \rightarrow \infty} u_{j} \leq u$ and $\lim _{j \rightarrow \infty} \int_{X}\left(u_{j}-u\right) \omega_{u_{j}}^{n}=0$.

Proof. Set

$$
M=\max \left(1, \sup _{j \geq 1}\left\|u_{j}\right\|_{L^{\infty}(X)},\|u\|_{L^{\infty}(X)}\right)<+\infty .
$$

i) $\Rightarrow$ ii). Given $\delta>0$, we have

$$
\begin{aligned}
\left|\int_{X}\left(u_{j}-u\right) \omega_{u_{j}}^{n}\right| & =\left|\int_{\left\{\left|u_{j}-u\right|<\delta\right\}}\left(u_{j}-u\right) \omega_{u_{j}}^{n}+\int_{\left\{\left|u_{j}-u\right| \geq \delta\right\}}\left(u_{j}-u\right) \omega_{u_{j}}^{n}\right| \\
& \leq \delta \int_{X} \omega_{u_{j}}^{n}+2 M \int_{\left\{\left|u_{j}-u\right| \geq \delta\right\}} \omega_{u_{j}}^{n} \\
& \leq \delta+(2 M)^{n+1} C_{X}\left(\left\{\left|u_{j}-u\right| \geq \delta\right\}\right) .
\end{aligned}
$$


It follows that

$$
\varlimsup_{j \rightarrow \infty}\left|\int_{X}\left(u_{j}-u\right) \omega_{u_{j}}^{n}\right| \leq \delta
$$

Therefore

$$
\varlimsup_{j \rightarrow \infty}\left|\int_{X}\left(u_{j}-u\right) \omega_{u_{j}}^{n}\right|=0 .
$$

Since $u_{j} \rightarrow u$ in $C_{X}$, it is easy to check that $\overline{\lim }_{j \rightarrow \infty} u_{j} \leq u$.

ii) $\Rightarrow$ i) In order to prove ii) $\Rightarrow$ i) we need two lemmas.

2.2. Lemma. Let $u, v \in P S H \cap L^{\infty}(X, \omega)$ be bounded. Then

$$
\left|\int_{X} d(u-v) \wedge d^{c}(u-v) \wedge \omega_{\varphi_{1}} \wedge \ldots \wedge \omega_{\varphi_{n-1}}\right| \leq C\left(\int_{X}(v-u)\left(\omega_{u}^{n}-\omega_{v}^{n}\right)\right)^{2^{1-n}}
$$

$\forall \varphi_{1}, \ldots, \varphi_{n-1} \in \operatorname{PSH}(X, \omega),-1 \leq \varphi_{1}, \ldots, \varphi_{n-1} \leq 0$, where $C$ is a positive constant depending only on $n$ and $\|u\|_{L^{\infty}(X)}\|v\|_{L^{\infty}(X)}$.

Proof. As in [B] we set

$$
\begin{aligned}
f & =u-v \\
a & =\int_{X}(v-u)\left(\omega_{u}^{n}-\omega_{v}^{n}\right) \\
& =\int_{X}(v-u) d d^{c}(u-v) \wedge\left(\sum_{j=0}^{n-1} \omega_{u}^{j} \wedge \omega_{v}^{n-1-j}\right) \\
& =\int_{X} d f \wedge d^{c} f \wedge T,
\end{aligned}
$$

where

$$
T=\sum_{j=0}^{n-1} \omega_{u}^{j} \wedge \omega_{v}^{n-1-j}
$$

For each $k=0, \ldots, n-1$ we will prove inductively that

$$
\int_{X} d f \wedge d^{c} f \wedge \omega_{u}^{i} \wedge \omega_{v}^{j} \wedge \omega_{\varphi_{1}} \wedge \ldots \wedge \omega_{\varphi_{k}} \leq C a^{2^{-k}}
$$

$\forall i, j: i+j+k=n-1$.

If $k=0$, then

$$
\int_{X} d f \wedge d^{c} f \wedge \omega_{u}^{i} \wedge \omega_{v}^{j} \leq \int_{X} d f \wedge d^{c} f \wedge T=a .
$$

Assume that (1) holds for $k-1$. We prove by induction on $t$ that

$$
\int_{X} d f \wedge d^{c} f \wedge \omega_{u}^{i} \wedge \omega_{v}^{j} \wedge \omega_{\varphi_{1}} \wedge \ldots \wedge \omega_{\varphi_{t}} \wedge \omega^{k-t} \leq C a^{2^{-k}}
$$

For $t=0,(2)$ holds by Theorem 2 in $[\mathrm{Bl}]$. Set

$$
S=\omega_{\varphi_{1}} \wedge \ldots \wedge \omega_{\varphi_{t-1}} \wedge \omega^{k-t} .
$$


We have

$$
\begin{aligned}
\int_{X} d f & \wedge d^{c} f \wedge \omega_{u}^{i} \wedge \omega_{v}^{j} \wedge \omega_{\varphi_{t}} \wedge S \\
& =\int_{X} d f \wedge d^{c} f \wedge \omega_{u}^{i} \wedge \omega_{v}^{j} \wedge \omega \wedge S+\int_{X} d f \wedge d^{c} f \wedge \omega_{u}^{i} \wedge \omega_{v}^{j} \wedge d d^{c} \varphi_{t} \wedge S .
\end{aligned}
$$

Since (2) holds for $t-1$, we only prove that

$$
\left|\int_{X} d f \wedge d^{c} f \wedge \omega_{u}^{i} \wedge \omega_{v}^{j} \wedge d d^{c} \varphi_{t} \wedge S\right| \leq C a^{2^{-k}} .
$$

Indeed, by integration by parts we have

$$
\begin{aligned}
\left|\int_{X} d f \wedge d^{c} f \wedge \omega_{u}^{i} \wedge \omega_{v}^{j} \wedge d d^{c} \varphi_{t} \wedge S\right| \\
\quad=\left|\int_{X} d^{c} \varphi_{t} \wedge d f \wedge d d^{c} f \wedge \omega_{u}^{i} \wedge \omega_{v}^{j} \wedge S\right| \\
=\left|\int_{X} d f \wedge d^{c} \varphi_{t} \wedge d d^{c} f \wedge \omega_{u}^{i} \wedge \omega_{v}^{j} \wedge S\right| \\
\leq\left|\int_{X} d f \wedge d^{c} \varphi_{t} \wedge \omega_{u} \wedge \omega_{u}^{i} \wedge \omega_{v}^{j} \wedge S\right|+\left|\int_{X} d f \wedge d^{c} \varphi_{t} \wedge \omega_{v} \wedge \omega_{u}^{i} \wedge \omega_{v}^{j} \wedge S\right| \\
=\left|\int_{X} d f \wedge d^{c} \varphi_{t} \wedge \omega_{u}^{i+1} \wedge \omega_{v}^{j} \wedge S\right|+\left|\int_{X} d f \wedge d^{c} \varphi_{t} \wedge \omega_{u}^{i} \wedge \omega_{v}^{j+1} \wedge S\right| .
\end{aligned}
$$

By the Schwarz inequality it follows that

$$
\begin{aligned}
\left|\int_{X} d f \wedge d^{c} \varphi_{t} \wedge \omega_{u}^{i+1} \wedge \omega_{v}^{j} \wedge S\right|^{2} \\
\leq \int_{X} d f \wedge d^{c} f \wedge \omega_{u}^{i+1} \wedge \omega_{v}^{j} \wedge S \int_{X} d \varphi_{t} \wedge d^{c} \varphi_{t} \wedge \omega_{u}^{i+1} \wedge \omega_{v}^{j} \wedge S \\
\quad=\int_{X} d f \wedge d^{c} f \wedge \omega_{u}^{i+1} \wedge \omega_{v}^{j} \wedge S \int_{X}-\varphi_{t} d d^{c} \varphi_{t} \wedge \omega_{u}^{i+1} \wedge \omega_{v}^{j} \wedge S \\
\leq \int_{X} d f \wedge d^{c} f \wedge \omega_{u}^{i+1} \wedge \omega_{v}^{j} \wedge S \int_{X}-\varphi_{t} \omega_{\varphi_{t}} \wedge \omega_{u}^{i+1} \wedge \omega_{v}^{j} \wedge S \\
\leq \int_{X} d f \wedge d^{c} f \wedge \omega_{u}^{i+1} \wedge \omega_{v}^{j} \wedge S \int_{X} \omega_{\varphi_{t}} \wedge \omega_{u}^{i+1} \wedge \omega_{v}^{j} \wedge S \\
=\int_{X} d f \wedge d^{c} f \wedge \omega_{u}^{i+1} \wedge \omega_{v}^{j} \wedge S \\
\leq C a^{2^{1-k}}
\end{aligned}
$$

(because (1) holds for $k-1$ ). 
Therefore

$$
\left|\int_{X} d f \wedge d^{c} \varphi_{t} \wedge \omega_{u}^{i+1} \wedge \omega_{v}^{j} \wedge S\right| \leq C a^{2^{-k}}
$$

Similarly

$$
\left|\int_{X} d f \wedge d^{c} \varphi_{t} \wedge \omega_{u}^{i} \wedge \omega_{v}^{j+1} \wedge S\right| \leq C a^{2^{-k}} .
$$

2.3. Lemma. Let $u_{j}, u \in P S H(X, \omega)$ be uniformly bounded. Then the following are equivalent:

i) $u_{j} \rightarrow u$ in $C_{X}$,

ii) $\lim _{j \rightarrow \infty} u_{j} \leq u$ and $\lim _{j \rightarrow \infty} \int_{X}\left(\tilde{u}_{j}-u_{j}\right) \omega_{u_{j}}^{n}=0$,

where $\tilde{u}_{j}=\max \left(u_{j}, u\right)$.

Proof. i) $\Rightarrow$ ii). This is the same as in i) $\Rightarrow$ ii) of Theorem 2.1.

ii) $\Rightarrow$ i). Since $\tilde{u}_{j} \rightarrow u$ and $\tilde{u}_{j}=\max \left(u_{j}, u\right)$, it is easy to see that $\tilde{u}_{j} \rightarrow u$ in $C_{X}$. Thus to prove $u_{j} \rightarrow u$ in $C_{X}$, it suffices to show that $\tilde{u}_{j}-u_{j} \rightarrow 0$ in $C_{X}$. Indeed, for every $\delta>0$ we have

$$
\begin{aligned}
C_{X}\left(\left\{\tilde{u}_{j}-u_{j}>\delta\right\}\right) & =\sup \left\{\int_{\left\{\tilde{u}_{j}-u_{j}>\delta\right\}} \omega_{\varphi}^{n}: \varphi \in \operatorname{PSH}(X, \omega),-1 \leq \varphi \leq 0\right\} \\
& \leq \frac{1}{\delta} \sup \left\{\int_{X}\left(\tilde{u}_{j}-u_{j}\right) \omega_{\varphi}^{n}: \varphi \in \operatorname{PSH}(X, \omega),-1 \leq \varphi \leq 0\right\} .
\end{aligned}
$$

In order to prove the lemma we prove by induction on $k=0, \ldots, n$ that

$$
\sup \left\{\int_{X}\left(\tilde{u}_{j}-u_{j}\right) \omega_{\varphi}^{k} \wedge \omega^{n-k}: \varphi \in \operatorname{PSH}(X, \omega),-1 \leq \varphi \leq 0\right\} \rightarrow 0
$$

as $j \rightarrow \infty$.

We show that (1) holds for $k=0$. We assume conversely that

$$
\sup \left\{\int_{X}\left(\tilde{u}_{j}-u_{j}\right) \wedge \omega^{n}: \varphi \in \operatorname{PSH}(X, \omega),-1 \leq \varphi \leq 0\right\} \nrightarrow \rightarrow 0
$$

as $j \rightarrow \infty$. We may assume that

$$
\int_{X}\left(\tilde{u}_{j}-u_{j}\right) \omega^{n} \geq \epsilon_{0}, \forall j \geq 1
$$

for some $\epsilon_{0}>0$. By [Ho, we also may assume that $u_{j} \rightarrow v \in \operatorname{PSH}(X, \omega)$ as $j \rightarrow \infty$ in $L^{1}(X)$ with $v \leq u$. Since $\tilde{u}_{j}-u_{j} \rightarrow u-v$ weakly, it follows that $D\left(\tilde{u}_{j}-u_{j}\right) \rightarrow D(u-v)$ weakly as $j \rightarrow \infty$ where $D u=\left(\frac{\partial u}{\partial z_{1}}, \ldots, \frac{\partial u}{\partial z_{n}}, \frac{\partial u}{\partial \bar{z}_{1}}, \ldots, \frac{\partial u}{\partial z_{n}}\right)$. From Lemma 2.2 we have

$$
\begin{aligned}
\int_{X}\left|D\left(\tilde{u}_{j}-u_{j}\right)\right|^{2} \omega^{n} & =\left|\int_{X} d\left(\tilde{u}_{j}-u_{j}\right) \wedge d^{c}\left(\tilde{u}_{j}-u_{j}\right) \omega^{n-1}\right| \\
& \leq C\left(\int_{X}\left(\tilde{u}_{j}-u_{j}\right)\left(\omega_{u_{j}}^{n}-\omega_{\tilde{u}_{j}}^{n}\right)\right)^{2^{1-n}} \\
& \leq C\left(\int_{X}\left(\tilde{u}_{j}-u_{j}\right) \omega_{u_{j}}^{n}\right)^{2^{1-n}} \rightarrow 0
\end{aligned}
$$


as $j \rightarrow \infty$. Combining this with the weak convergence of $D\left(\tilde{u}_{j}-u_{j}\right)$ to $D(u-v)$ we have $D(u-v)=0$. Hence $u-v=c \geq 0$ a.e in $X$. Since $u$ and $v$ are $\omega$-psh, we have $u-v=c$ on $X$. We show that $c=0$. Indeed, we have

$$
\begin{aligned}
\int_{X}\left(\tilde{u}_{j}-u_{j}\right) \omega_{u_{j}}^{n} & \geq \int_{X}\left(u-u_{j}\right) \omega_{u_{j}}^{n} \\
& =c \int_{X} \omega^{n}+\int_{X}\left(v-u_{j}\right) \omega_{u_{j}}^{n} \\
& =c+\int_{X}\left(v-u_{j}\right) \omega_{u_{j}}^{n} .
\end{aligned}
$$

Given $\epsilon>0$, by BT2] we find an open subset $G$ of $X$ with $C_{X}(G)<\epsilon$ and $j_{0}$ such that

$$
u_{j}(z) \leq v(z)+\epsilon, \forall j \geq j_{0}, z \in X \backslash G
$$

It follows that

$$
\begin{aligned}
\int_{X}\left(v-u_{j}\right) \omega_{u_{j}}^{n} & \geq-M^{n+1} C_{X}(G)-\epsilon \int_{X} \omega_{u_{j}}^{n} \\
& \geq-M^{n+1} \epsilon-\epsilon
\end{aligned}
$$

for $j \geq j_{0}$. Letting $j \rightarrow \infty$ and $\epsilon \rightarrow 0$ we obtain

$$
\varlimsup_{j \rightarrow \infty} \int_{X}\left(v-u_{j}\right) \omega_{u_{j}}^{n} \geq 0 .
$$

There from $i i$ ) we have

$$
0=\varlimsup_{j \rightarrow \infty} \int_{X}\left(\tilde{u}_{j}-u_{j}\right) \omega_{u_{j}}^{n} \geq c \geq 0 .
$$

Thus $c=0$ and $u=v$. This means that $\tilde{u}_{j}$ and $u_{j} \rightarrow u$ in $L^{1}(X)$, which contradicts (2).

Assume that (1) holds for $k-1$. For each $\varphi \in \operatorname{PSH}(X, \omega),-1 \leq \varphi \leq 0$, we have

$$
\begin{aligned}
\int_{X}\left(\tilde{u}_{j}-u_{j}\right) \omega_{\varphi}^{k} \wedge \omega^{n-k}= & \int_{X}\left(\tilde{u}_{j}-u_{j}\right) \omega_{\varphi}^{k-1} \wedge \omega^{n-k+1} \\
& +\int_{X}\left(\tilde{u}_{j}-u_{j}\right) d d^{c} \varphi \wedge \omega_{\varphi}^{k-1} \wedge \omega^{n-k} \\
= & \int_{X}\left(\tilde{u}_{j}-u_{j}\right) \omega_{\varphi}^{k-1} \wedge \omega^{n-k+1} \\
& -\int_{X} d\left(\tilde{u}_{j}-u_{j}\right) \wedge d^{c} \varphi \wedge \omega_{\varphi}^{k-1} \wedge \omega^{n-k} .
\end{aligned}
$$


By the induction hypothesis it remains to prove that

$$
\sup \left\{\left|\int_{X} d\left(\tilde{u}_{j}-u_{j}\right) \wedge d^{c} \varphi \wedge \omega_{\varphi}^{k-1} \wedge \omega^{n-k}\right|: \varphi \in \operatorname{PSH}(X, \omega),-1 \leq \varphi \leq 0\right\} \rightarrow 0
$$

as $j \rightarrow \infty$. Indeed, by the Schwarz inequality, we have

$$
\begin{array}{rl}
\mid \int_{X} d & \left.d\left(\tilde{u}_{j}-u_{j}\right) \wedge d^{c} \varphi \wedge \omega_{\varphi}^{k-1} \wedge \omega^{n-k}\right|^{2} \\
& \leq \int_{X} d \varphi \wedge d^{c} \varphi \wedge \omega_{\varphi}^{k-1} \wedge \omega^{n-k} \int_{X} d\left(\tilde{u}_{j}-u_{j}\right) \wedge d^{c}\left(\tilde{u}_{j}-u_{j}\right) \wedge \omega_{\varphi}^{k-1} \wedge \omega^{n-k} \\
& =\int_{X}-\varphi d d^{c} \varphi \wedge \omega_{\varphi}^{k-1} \wedge \omega^{n-k} \int_{X} d\left(\tilde{u}_{j}-u_{j}\right) \wedge d^{c}\left(\tilde{u}_{j}-u_{j}\right) \wedge \omega_{\varphi}^{k-1} \wedge \omega^{n-k} \\
& \leq \int_{X}-\varphi \omega_{\varphi}^{k} \wedge \omega^{n-k} \int_{X} d\left(\tilde{u}_{j}-u_{j}\right) \wedge d^{c}\left(\tilde{u}_{j}-u_{j}\right) \wedge \omega_{\varphi}^{k-1} \wedge \omega^{n-k} \\
\leq & \int_{X} \omega_{\varphi}^{k} \wedge \omega^{n-k} \int_{X} d\left(\tilde{u}_{j}-u_{j}\right) \wedge d^{c}\left(\tilde{u}_{j}-u_{j}\right) \wedge \omega_{\varphi}^{k-1} \wedge \omega^{n-k} \\
& =\int_{X} d\left(\tilde{u}_{j}-u_{j}\right) \wedge d^{c}\left(\tilde{u}_{j}-u_{j}\right) \wedge \omega_{\varphi}^{k-1} \wedge \omega^{n-k}
\end{array}
$$

(by Lemma 2.2)

$$
\begin{aligned}
& \leq C\left(\int_{X}\left(\tilde{u}_{j}-u_{j}\right)\left(\omega_{u_{j}}^{n}-\omega_{\tilde{u}_{j}}^{n}\right)\right)^{2^{1-n}} \\
& \leq C\left(\int_{X}\left(\tilde{u}_{j}-u_{j}\right) \omega_{u_{j}}^{n}\right)^{2^{1-n}} \rightarrow 0
\end{aligned}
$$

as $j \rightarrow \infty$.

Now we can complete the proof of ii) $\Rightarrow$ i) in Theorem 2.1. By Lemma 2.3 it remains to show that

$$
\lim _{j \rightarrow \infty} \int_{X}\left(\tilde{u}_{j}-u_{j}\right) \omega_{u_{j}}^{n}=0
$$

The equality follows from the hypothesis $i i$ ) and the convergence of $\tilde{u}_{j}$ to $u$ in $C_{X}$.

2.4. Theorem. Let $X$ be a compact Kahler manifold and $S$ a smooth hypersurface in $X$. Let $u_{j}, u \in P S H(X, \omega)$ be uniformly bounded such that $u_{j} \rightarrow u$ in $C_{X}$ and $\operatorname{supp} \omega_{u_{j}}^{n} \subset K \Subset X \backslash S$ for $j \geq 1$. Then $\left.\left.u_{j}\right|_{S} \rightarrow u\right|_{S}$ in $C_{S}$ as $j \rightarrow \infty$.

Proof. Let $\left\{U_{i}\right\}_{i=1, \ldots, m}$ be an open cover of $X$ satisfying

i) For each $i=1, \ldots, m$, there exists a holomorphic function $f_{i}$ on a neighbourhood of $\bar{U}_{i}$ such that $S \cap U_{i}=\left\{f_{i}=0\right\}, f_{i}^{\prime}(z) \neq 0$ for $z \in \bar{U}_{i}$ and $\left\|f_{i}\right\|_{L^{\infty}\left(U_{i}\right)} \leq 1$.

ii) For each $i=1, \ldots, m$ either $U_{i} \cap K=\emptyset$ or $U_{i} \cap S=\emptyset$. 
Let $\left\{\varphi_{i}\right\}_{i=1, \ldots, m}$ be a $C^{\infty}$-partition of unity associated with $\left\{U_{i}\right\}_{i=1, \ldots, m}$. Set $\psi_{i}=\log \left|f_{i}\right|, \forall i=1, \ldots, m$ and $\tilde{u}_{j}=\max \left(u_{j}, u\right), \forall j \geq 1$. Since $u_{j} \rightarrow u$ in $C_{X}$, we have $\varlimsup_{j \rightarrow \infty} u_{j} \leq u$ in $X$ and hence $\varlimsup_{j \rightarrow \infty} u_{j} \leq u$ in $S$. By Lemma 2.3 it remains to show that

$$
\varlimsup_{j \rightarrow \infty} \int_{S}\left(\tilde{u}_{j}-u_{j}\right) \omega_{\left.u_{j}\right|_{S}}^{n-1} \leq 0
$$

Indeed, we have by Corollary 4.2 in [BT3,

$$
\begin{aligned}
& \int_{S}\left(\tilde{u}_{j}-u_{j}\right) \omega_{\left.u_{j}\right|_{S}}^{n-1} \\
& =\sum_{i=1}^{m} \int_{S} \varphi_{i}\left(\tilde{u}_{j}-u_{j}\right) \omega_{\left.u_{j}\right|_{S}}^{n-1} \\
& =\sum_{i=1}^{m} \int_{S \cap U_{i}} \varphi_{i}\left(\tilde{u}_{j}-u_{j}\right) \omega_{u_{j} \mid S}^{n-1} \\
& =\frac{1}{2 \pi} \sum_{i=1}^{m} \int_{U_{i}} \varphi_{i}\left(\tilde{u}_{j}-u_{j}\right) d d^{c} \psi_{i} \wedge \omega_{u_{j}}^{n-1} \\
& =\frac{1}{2 \pi} \sum_{i=1}^{m} \int_{X} \varphi_{i}\left(\tilde{u}_{j}-u_{j}\right) d d^{c} \psi_{i} \wedge \omega_{u_{j}}^{n-1} \\
& =-\frac{1}{2 \pi} \sum_{i=1}^{m} \int_{X}\left(\tilde{u}_{j}-u_{j}\right) d \varphi_{i} \wedge d^{c} \psi_{i} \wedge \omega_{u_{j}}^{n-1} \\
& -\frac{1}{2 \pi} \sum_{i=1}^{m} \int_{X} \varphi_{i} d\left(\tilde{u}_{j}-u_{j}\right) \wedge d^{c} \psi_{i} \wedge \omega_{u_{j}}^{n-1} \\
& =-\frac{1}{2 \pi} \sum_{i=1}^{m} \int_{X}\left(\tilde{u}_{j}-u_{j}\right) d \varphi_{i} \wedge d^{c} \psi_{i} \wedge \omega_{u_{j}}^{n-1} \\
& +\frac{1}{2 \pi} \sum_{i=1}^{m} \int_{X} \varphi_{i} d \psi_{i} \wedge d^{c}\left(u_{j}-\tilde{u}_{j}\right) \wedge \omega_{u_{j}}^{n-1} \\
& =-\frac{1}{2 \pi} \sum_{i=1}^{m} \int_{X}\left(\tilde{u}_{j}-u_{j}\right) d \varphi_{i} \wedge d^{c} \psi_{i} \wedge \omega_{u_{j}}^{n-1} \\
& -\frac{1}{2 \pi} \sum_{i=1}^{m} \int_{X} \psi_{i} d \varphi_{i} \wedge d^{c}\left(u_{j}-\tilde{u}_{j}\right) \wedge \omega_{u_{j}}^{n-1} \\
& -\frac{1}{2 \pi} \sum_{i=1}^{m} \int_{X} \varphi_{i} \psi_{i} d d^{c}\left(u_{j}-\tilde{u}_{j}\right) \wedge \omega_{u_{j}}^{n-1} \\
& =A_{j}+B_{j}+C_{j} \text {. }
\end{aligned}
$$


For $C_{j}$ we have

$$
\begin{aligned}
C_{j} & =-\frac{1}{2 \pi} \sum_{i=1}^{m} \int_{X} \varphi_{i} \psi_{i} d d^{c}\left(u_{j}-\tilde{u}_{j}\right) \wedge \omega_{u_{j}}^{n-1} \\
& =-\frac{1}{2 \pi} \sum_{i=1}^{m} \int_{X} \varphi_{i} \psi_{i}\left(\omega_{u_{j}}-\omega_{\tilde{u}_{j}}\right) \wedge \omega_{u_{j}}^{n-1} \\
& \leq-\frac{1}{2 \pi} \sum_{i=1}^{m} \int_{X} \varphi_{i} \psi_{i} \omega_{u_{j}}^{n}=0
\end{aligned}
$$

(because supp $\omega_{u_{j}}^{n} \subset K$ for $j \geq 1$ and either $U_{i} \cap K=\emptyset$ or $U_{i} \cap S=\emptyset$ for $i=1, \ldots, m)$. Next write

$$
\begin{aligned}
B_{j} & =-\frac{1}{2 \pi} \sum_{i=1}^{m} \int_{X} \psi_{i} d \varphi_{i} \wedge d^{c}\left(u_{j}-\tilde{u}_{j}\right) \wedge \omega_{u_{j}}^{n-1} \\
& =-\frac{1}{2 \pi} \sum_{i=1}^{m} \int_{X} \psi_{i} d\left(u_{j}-\tilde{u}_{j}\right) \wedge d^{c} \varphi_{i} \wedge \omega_{u_{j}}^{n-1} \\
& =-\frac{1}{2 \pi} \int_{X} d\left(u_{j}-\tilde{u}_{j}\right) \wedge\left(\sum_{i=1}^{m} \psi_{i} d^{c} \varphi_{i}\right) \wedge \omega_{u_{j}}^{n-1} .
\end{aligned}
$$

Obviously $g=\sum_{i=1}^{m} \psi_{i} d^{c} \varphi_{i}$ is smooth. Indeed, let $z \in X$. We can assume that $\left\{i=1, \ldots, m: z \in U_{i}\right\}=\{1, \ldots, k\}$. Take a neighbourhood $V$ of $z$ such that $V \subset U_{i}$ for $i=1, \ldots, k$ and $V \cap \operatorname{supp} \varphi_{i}=\emptyset$ for $i=k+1, \ldots, m$. On $V$ we have

$$
\begin{aligned}
\sum_{i=1}^{m} \psi_{i} d^{c} \varphi_{i} & =\sum_{i=2}^{m}\left(\psi_{i}-\psi_{1}\right) d^{c} \varphi_{i} \\
& =\sum_{i=2}^{k}\left(\psi_{i}-\psi_{1}\right) d^{c} \varphi_{i} \\
& =\sum_{i=2}^{k}\left(\log \frac{\left|f_{i}\right|}{\left|f_{1}\right|}\right) d^{c} \varphi_{i}
\end{aligned}
$$

Therefore $g$ is smooth. Thus for $B_{j}$ we have

$$
\begin{aligned}
\left|B_{j}\right| & =\left|\int_{X} d\left(u_{j}-\tilde{u}_{j}\right) \wedge g \wedge \omega_{u_{j}}^{n-1}\right| \\
& =\left|\int_{X}\left(\tilde{u}_{j}-u_{j}\right) d g \wedge \omega_{u_{j}}^{n-1}\right| \leq C \int_{X}\left(\tilde{u}_{j}-u_{j}\right) \omega \wedge \omega_{u_{j}}^{n-1},
\end{aligned}
$$

where $C$ is a positive constant independent on $g$. Since $\tilde{u}_{j}$ and $u_{j} \rightarrow u$ in $C_{X}$, it follows that $B_{j} \rightarrow 0$ as $j \rightarrow \infty$.

Similarly as above, $h=\sum_{i=1}^{m} d \varphi_{i} \wedge d^{c} \psi_{i}$ is smooth. Thus we can find $C>0$ such that

$$
\left|A_{j}\right| \leq C \int_{X}\left(\tilde{u}_{j}-u_{j}\right) \omega \wedge \omega_{u_{j}}^{n-1} \rightarrow 0
$$

as $j \rightarrow \infty$. 
From Theorem 2.4 we obtain the following.

2.5. Corollary. Let $X$ and $S$ be as in Theorem 2.4 and $u_{j}, u \in P S H(X, \omega)$ such that $u_{j}$ increases to $u$ a.e. on $X$ and $\operatorname{supp} \omega_{u_{j}}^{n} \subset K \Subset X \backslash S$ for $j \geq 1$. Then $\left.u_{j}\right|_{S}$ increases to $\left.u\right|_{S}$ a.e. on $S$.

Remark. Corollary 2.5 was proved by Bedford and Taylor in BT3 for $X=\mathbf{C} P^{n}$.

\section{Some Applications}

In this section we apply the results obtained in Section 2 to investigate global extremal functions and $\omega$-plurisubharmonic hulls of $\omega$-pluripolar sets in a compact Kahler manifold $X$.

Given $E$ a subset of $X$ and $Q$ a function on $E$, define

$$
V_{E, Q}=\sup \{\varphi \in \operatorname{PSH}(X, \omega): \varphi \leq Q \text { on } E\} .
$$

$V_{E, Q}$ is called the global extremal function of $E$ with the weight $Q$. We write $V_{E}=V_{E, 0}$.

3.1. Theorem. Let $X$ be a compact Kahler manifold and $S$ a smooth hypersurface in $X$. Let $K$ be a compact set in $X \backslash S$ and $Q$ be a lower semicontinuous function on $K$. Then

$$
\left(\left.V_{K, Q}\right|_{S}\right)^{*}=\left.V_{K, Q}^{*}\right|_{S}
$$

We need the following.

3.2. Lemma. Let $K$ be a compact set in $X$ and $\left\{Q_{j}\right\}$ be a sequence of lower semicontinuous functions on $K$ increasing to $Q$. Then $\left\{V_{K, Q_{j}}\right\}$ increases to $V_{K, Q}$.

Proof. Let $\varphi \in \operatorname{PSH}(X, \omega), \varphi \leq Q$ on $K$. Since $\varphi-Q_{j} \searrow \varphi-Q \leq 0$ on $K$, by Dini's theorem for every $\epsilon>0$ there exists $j_{0}$ such that $\varphi-Q_{j} \leq \epsilon$ on $K$ for $j \geq j_{0}$. This implies that $\varphi-\epsilon \leq V_{K, Q_{j}}$ for $j \geq j_{0}$. It follows that $V_{K, Q} \leq \lim _{j \rightarrow \infty} V_{K, Q_{j}}$. Therefore $\lim _{j \rightarrow \infty} V_{K, Q_{j}}=V_{K, Q}$ because obviously $\lim _{j \rightarrow \infty} V_{K, Q_{j}} \leq V_{K, Q}$.

Now we continue the proof of Theorem 3.1. Take a compact $\epsilon$-neighbourhood $E$ of $K$ with $E \subset X \backslash S$ and a sequence $Q_{j}$ of continuous function on $E$ such that $Q_{j} \nearrow Q$, where we define $Q=+\infty$ on $E \backslash K$. As in [Si, $V_{E, Q_{j}}$ is $\omega$-psh continuous and moreover $\operatorname{supp} \omega_{V_{E, Q_{j}}}^{n} \subset E \Subset X \backslash S$ for $j \geq 1$. By Lemma 3.2, $V_{E, Q_{j}}$ increases to $V_{E, Q}^{*}$ a.e. on $X$. Corollary 2.5 implies that $V_{E, Q_{j}}$ increases to $V_{E, Q}^{*}$ a.e. on $S$. Therefore we have

$$
V_{E, Q}=\lim _{j \rightarrow \infty} V_{E, Q_{j}}=V_{E, Q}^{*}
$$

a.e. on $S$. It follows that

$$
\left(\left.V_{E, Q}\right|_{S}\right)^{*} \geq\left. V_{E, Q}^{*}\right|_{S}
$$

a.e. on $S$. Since both functions are $\omega_{S}$-psh on $S$ we have

$$
\left(\left.V_{E, Q}\right|_{S}\right)^{*} \geq\left. V_{E, Q}^{*}\right|_{S} .
$$

Therefore

because obviously

$$
\left(\left.V_{E, Q}\right|_{S}\right)^{*}=\left.V_{E, Q}^{*}\right|_{S}
$$

$$
\left(\left.V_{E, Q}\right|_{S}\right)^{*} \leq\left. V_{E, Q}^{*}\right|_{S} .
$$

Let $\mathcal{L}\left(\mathbf{C}^{n}\right)$ be the family of plurisubharmonic functions on $\mathbf{C}^{n}$ that satisfy

$$
\varphi(z) \leq \frac{1}{2} \log \left(1+|z|^{2}\right)+C_{\varphi}, z \in \mathbf{C}^{n} .
$$


We consider a 1-to-1 correspondence between $\operatorname{PSH}\left(\mathbf{C} P^{n}, \omega_{\mathbf{C} P^{n}}\right)$ and the homogeneous Lelong class

$$
\mathcal{H}\left(\mathbf{C}^{n+1}\right)=\left\{\varphi \in \mathcal{L}\left(\mathbf{C}^{n+1}\right): \varphi(t z)=\varphi(z)+\log |t|, z \in \mathbf{C}^{n+1}, t \in \mathbf{C}\right\},
$$

which is given by the natural mapping

$$
\varphi \in \mathcal{H}\left(\mathbf{C}^{n+1}\right) \rightarrow \tilde{\varphi}(z)=\varphi(z)-\log |z|, z \in \mathbf{C}^{n+1} .
$$

From the 1-to-1 mapping and Theorem 3.1 we generalize Theorem 1.1 in [Ko1].

3.3. Corollary. Let $K$ be a compact subset in $\mathbf{C}^{n}$ and $Q$ be a lower semicontinuous function on $K$. Then

$$
\varlimsup_{(t, \xi) \rightarrow(0, z)} \psi_{1 \times K, Q}(t, \xi)=\varlimsup_{\xi \rightarrow z} \psi_{1 \times K, Q}(0, \xi), z \in \mathbf{C}^{n}
$$

where

$$
\psi_{1 \times K, Q}(t, z)=\sup \left\{\varphi(t, z): \varphi \in \mathcal{H}\left(\mathbf{C}^{n+1}\right), \varphi(1, z) \leq Q(z) \text { on } K\right\} .
$$

3.4. Theorem. Let $X$ be a compact Kahler manifold and $S$ a smooth hypersurface in $X$. Let $E$ be an $\omega$-pluripolar subset in $X \backslash S$. Then $E_{X}^{*} \cap S$ is also $\omega_{S}$-pluripolar in $S$.

Proof. Take $v \in \operatorname{PSH}(X, \omega), v \not \equiv-\infty$ such that $E \subset\{v=-\infty\}$ and $v \leq-1$. Let $\Omega_{j}$ be an increasing sequence of smooth domains exhausting $X \backslash S$. For each $\epsilon>0$ and $j \geq 1$, set

$$
u_{\epsilon, j}=\sup \left\{\varphi \in \operatorname{PSH}(X, \omega): \varphi \leq \max \left(\epsilon v,-2^{j}\right) \text { on } \Omega_{j}\right\} .
$$

It is easy to see that for each $j \geq 1$,

$$
\max \left(\epsilon v,-2^{j}\right) \leq u_{\epsilon, j} \leq V_{\Omega_{j}}, \operatorname{supp} \omega_{u_{\epsilon, j}}^{n} \subset \bar{\Omega}_{j}
$$

and $u_{\epsilon, j} \nearrow V_{\Omega_{j}}$ a.e. on $X$ as $\epsilon \rightarrow 0$. By Corollary 2.5 it follows that $u_{\epsilon, j} \nearrow V_{\Omega_{j}} \geq 0$ on $S \backslash F_{j}$ as $\epsilon \rightarrow 0$, where $F_{j}$ is an $\omega_{S}$-pluripolar set in $S$. Take $z_{0} \in S \backslash\left(\bigcup_{j=1}^{\infty} F_{j}\right)$ and $\epsilon_{j}>0$ such that

$$
u_{\epsilon, j}\left(z_{0}\right) \geq-\frac{1}{2^{j}}
$$

for $j \geq 1$. Set

$$
u=\sum_{j=1}^{\infty} \frac{u_{\epsilon_{j}, j}}{2^{j}} .
$$

Then $u$ is $\omega$-psh on $X$ satisfying $u=-\infty$ on $E$. Moreover $u\left(z_{0}\right) \geq-1$. Thus $E_{X}^{*} \cap S$ is $\omega_{S}$-pluripolar in $S$. The theorem is proved.

\section{ACKNOWLEDGMENTS}

The author is grateful to Professor Nguyen Van Khue for suggesting the problem and for many helpful discussions during the preparation of this work. The author is also indebted to the referee for his useful comments that helped to improve the paper. 


\section{REFERENCES}

[Bl] Z. Blocki, Uniqueness and stability for the complex Monge-Ampère equation on compact Kahler manifolds, Indiana Univ. Math. J. 52 (2003), no. 6, 1697-1701. MR2021054 (2004m:32073)

[BT1] E. Bedford and B. A. Taylor, The Dirichlet problem for the complex Monge-Ampère operator, Invent. Math. 37 (1976), 1-44. MR0445006 (56:3351)

[BT2] E. Bedford and B. A. Taylor, A new capacity for plurisubharmonic functions, Acta Math. 149 (1982), 1-40. MR674165 (84d:32024)

[BT3] E. Bedford and B. A. Taylor, Plurisubhurmonic functions with logarithmic singularities, Ann. Inst. Fourier 38 (1988), 133-171. MR978244 (90f:32016)

[BT4] E. Bedford and B. A. Taylor, Uniqueness for the complex Monge-Ampère equation for functions of logarithmic growth, Indiana Univ. Math. J. 38 (1989), 455-469. MR997391 (90i:32025)

[Ce1] U. Cegrell, Pluricomplex energy, Acta Mathematica 180 (1998), 187-217. MR.1638768 (99h:32016)

[Ce2] U. Cegrell, The general definition of the complex Monge-Ampère operator, Ann. Inst. Fourier (Grenoble) 54 (2004), 159-179. MR 2069125 (2005d:32062)

[Ce3] U. Cegrell, Convergence in capacity, Technical report, Issac Newton Institute for Mathematical Sciences, 2001.

[GZ] V. Guedj and A. Zeriahi, Intrinsic capacities on compact Kahler manifolds, J. Geom. Anal. 15 (2005), no. 4, 607-639. MR2203165 (2006j:32041)

[Ho] L. Hörmander, Notions of Convexity, Progress in Mathematics 127, Birkhäuser, Boston, 1994. MR 1301332 (95k:00002)

[Ko1] S. Kołodziej, Capacities associated to the Siciak extremal function, Ann. Polon. Math. XLIX (1989), 279-290. MR997520 (90h:32039)

[Ko2] S. Kołodziej, The Monge-Ampère equation on compact Kahler manifolds, Indiana Univ. Math. J. 52 (2003), 667-686. MR1986892(2004i:32062)

[Si] J. Siciak, Extremal plurisubharmonic functions in $\mathbf{C}^{n}$, Ann. Polon. Math. XXXIX (1981), 175-210. MR617459 (83e:32018)

[Xi1] Y. Xing, Continuity of the complex Monge-Ampère operator, Proc. Amer. Math. Soc. 124 (1996), 457-467. MR1322940(96d:32015)

[Xi2] Y.Xing, Complex Monge-Ampère measures of pluriharmonic functions with bounded values near the boundary, Canad. J. Math. 52 (2000), 1085-1100. MR.1782339 (2001h:32070)

Department of Mathematics, University of Education (Dai hoc Su Pham Ha Noi), CauGiay, Hanoi, Vietnam

E-mail address: phhiep_vn@yahoo.com 